\title{
Host factors that impact the biodistribution and persistence of multipotent adult progenitor cells
}

\author{
Jakub Tolar, Matthew J. O’Shaughnessy, Angela Panoskaltsis-Mortari, Ron T. McElmurry, Scott Bell, Megan Riddle, R. Scott Mclvor, \\ Stephen R. Yant, Mark A. Kay, Diane Krause, Catherine M. Verfaillie, and Bruce R. Blazar
}

\begin{abstract}
Multipotent adult progenitor cells (MAPCs) are marrow-derived pluripotent stem cells with a broad differentiation potential. We sought to identify factors that affect adoptively transferred MAPCs. In vitro, MAPCs expressed low levels of major histocompatibility complex (MHC) antigens, failed to stimulate $\mathrm{CD}^{+}$and $\mathrm{CD}^{+}{ }^{+} \mathrm{T}$-cell alloresponses, and were targets of NK cytolysis. To study in vivo biodistribution, we labeled MAPCs with luciferase for sequential quantification of bioluminescence and DsRed2 for immunohistochemical analy-
\end{abstract}

sis. C57BL/6 MAPCs were infused intravenously into C57BL/6, Rag-2 ${ }^{-1-}$ (T- and B-cell-deficient), and Rag-2 $2^{-I-} / \mathrm{IL}-2 \mathrm{R} \gamma_{\mathrm{c}}{ }^{-/-}$ (T-, B-, and NK-cell-deficient) mice. In C57BL/6 mice, MAPCs were transiently detected only in the chest compared with long-term persistence in T- and B-celldeficient mice. NK depletion reduced MAPC elimination. Because the lungs were the major uptake site after intravenous injection, intra-arterial injections were tested and found to result in more widespread biodistribution. Widespread
MAPC biodistribution and long-term persistence were seen in irradiated recipients given allogeneic marrow and MAPCs; such MAPCs expressed MHC class I antigens in tissues. Our data indicate that the biodistribution and persistence of reporter gene-labeled MAPCs are maximized after intra-arterial delivery or host irradiation and that T cells, B cells, and NK cells contribute to in vivo MAPC rejection. (Blood. 2006;107:4182-4188)

() 2006 by The American Society of Hematology

\section{Introduction}

Multipotent adult progenitor cells (MAPCs) are a novel class of stem cells derived from adult tissues, including bone marrow, muscle, and brain. ${ }^{1-5}$ MAPCs are pluripotent, and a single MAPC injected into a blastocyst contributes to all tissues, including skeletal muscle, cardiac muscle, liver, lung, intestine, central nervous system, skin, spleen, blood, and marrow. ${ }^{2}$ Clonal MAPCs are able to differentiate in vitro into various lineages of mesodermal, ectodermal, and endodermal origin and contribute to terminally differentiated tissues when postnatally grafted. ${ }^{1-5}$ This capacity is enhanced during injury, ${ }^{2}$ suggesting a possible role for MAPCs in the regeneration of tissues injured by chemotherapy or radiation during conditioning for hematopoietic stem cell transplantation.

For any clinical application of MAPCs, it is critical to understand the mechanisms that can lead to immune rejection of the infused cells. Because undifferentiated MAPCs express low levels of major histocompatibility complex (MHC) class I, MAPCs may be ideal targets for NK cell-mediated killing. If so, NK-cell resistance may serve as a barrier to the infusion of undifferentiated MAPCs in vivo. As a part of innate immune defense, NK cells can kill infected or transformed tissues before sensitization and without restriction by MHC antigens. ${ }^{6-8}$ Because NK function can influence the outcome of hematopoietic cell transplantation, ${ }^{9,10}$ we reasoned that NK activity may have a role in MAPC homing and rejection.

Other important considerations in the use of MAPCs include understanding the host factors that might affect in vivo MAPC biodistribution and persistence. Therefore, we analyzed the role of MAPCs as targets of immune response as a function of engraftment and persistence of infused MAPCs using a 2-reporter gene system with red fluorescence protein derived from Discosoma coral $\left(\right.$ DsRed2) ${ }^{11}$ and firefly luciferase. ${ }^{12}$ Donor MAPC-derived optical signatures of fluorescent (DsRed2) and bioluminescent (luciferase) signals were chosen to complement each other in postmortem tissue analysis and in noninvasive imaging in live animals, respectively.

The success of stem cell therapies largely depends on immune tolerance of the infused cells in the host and on the proliferation and differentiation potential of the cellular graft. With the use of mice with various degrees of immune competence, we show for the first time that the depletion of NK cells in the recipient results in higher levels of donor MAPC engraftment and persistence in real time in vivo. When compared with intravenous delivery of the same dose of MAPCs, intra-arterial infusion resulted in more diverse
From the Pediatric Hematology/Oncology/Blood and Marrow Transplant Program, the Department of Genetics, Cell Biology and Development, and the Stem Cell Institute, University of Minnesota, Minneapolis; the Department of Pediatrics, Stanford University School of Medicine, Stanford, CA; and the Department of Laboratory Medicine, Yale University School of Medicine, New Haven, CT.

Submitted August 12, 2005; accepted January 5, 2006. Prepublished online as Blood First Edition Paper, January 12, 2006; DOI 10.1182/blood-2005-08-3289.

Supported in part by the Children's Cancer Research Fund (J.T.), the American Society of Clinical Oncology (ASCO) Young Investigator Award (J.T.), the Fanconi Anemia Research Fund (J.T., B.R.B.), Athersys (B.R.B.), and National Institutes of Health grants Child Health Research Scholar Award 5K12-HD033692-10 (J.T.), DK49022 (M.A.K.), RO1 HL55209 (B.R.B.), RO1 HL52952, and RO1 HL49997
(B.R.B.) and an NCRR Shared Instrumentation Grant (ISIORR16851).

J.T., M.J.O., and A.P.M. designed and performed research, analyzed data, and wrote the paper. R.T.M., S.B., and M.R. performed research and analyzed data. R.S.M., S.R.Y., M.A.K., D.K., and C.M.V. contributed vital new reagents and analytical tools. B.R.B. designed research and wrote the paper.

Reprints: Jakub Tolar, Pediatric Hematology/Oncology/Blood and Marrow Transplantation Program, MMC 366, 420 Delaware St SE, Minneapolis, MN 55455; e-mail: tolar003@umn.edu.

The publication costs of this article were defrayed in part by page charge payment. Therefore, and solely to indicate this fact, this article is hereby marked "advertisement" in accordance with 18 U.S.C. section 1734.

(C) 2006 by The American Society of Hematology 
homing of MAPCs and in higher levels of persistent engraftment. Additionally, we observed that conditioning for bone marrow transplantation using high-dose total body irradiation overcomes MAPC rejection. Therefore, immune competence of the recipient, route of delivery, and conditioning regimen are likely to modulate the therapeutic impact of the MAPC infusion.

\section{Materials and methods}

\section{Mouse strains}

C57BL/6 (termed B6) $\left(\mathrm{H} 2^{\mathrm{b}}\right)$ and B10.BR $\left(\mathrm{H} 2^{\mathrm{k}}\right)$ mice were obtained from The Jackson Laboratory (Bar Harbor, ME). BALB/c ( $2^{2}$ d) mice were obtained from the National Institutes of Health (Hartford, CT). B6 recombinase activating gene 2-deficient $\left(\mathrm{Rag}-2^{-1-}\right)$ mice were obtained from Taconic Farms (Germantown, NY). B6 mice carrying mutations in Rag-2 and the common cytokine receptor ( $\operatorname{Rag} 2 / \mathrm{IL}-2 \mathrm{R} \gamma \mathrm{c}^{-/-}$) were a gift from Dr Stephen Jameson (University of Minnesota, Minneapolis). All mice were housed under specific pathogen-free conditions, fed ad libitum according to the guidelines of the University of Minnesota Research Animal Resources, and used at 6 to 12 weeks of age. All protocols involving mice were approved by the Institutional Animal Care and Use Committee (IACUC).

\section{NK-cell depletion}

To deplete NK cells in vivo, we gave some mice injections of anti-NK1.1 monoclonal antibody (hybridoma PK136, rat $\mathrm{IgG}_{2 \mathrm{a}}$; provided by Dr Koo, Merck-Sharpe-Dohme, Rahway, NJ) 3 days before MAPC infusion and then twice a week for 30 days.

\section{Bone marrow transplantation}

B6 mice were lethally irradiated by $\mathrm{x}$-ray with $8.0 \mathrm{~Gy}$ on the day before transplantation with $20 \times 10^{6} \mathrm{~B} 10$.BR bone marrow (BM) cells with or without $10^{6}$ of C57BL/6 luciferase- and DsRed2-expressing MAPCs (MAPC DLs). BM was T cell depleted by incubation with anti-Thy1.2 antibody (30-H-12; National Cell Culture Center, Minneapolis, MN) and rabbit complement (Nieffengger, Woodland, CA).

\section{MAPC culture, labeling, and injection}

MAPCs were isolated from adult $129 \times \mathrm{C} 57 \mathrm{BL} / 6 \mathrm{~J} \mathrm{BM}\left(\mathrm{H}^{\mathrm{b}}\right.$, transgenic for LacZ and NeoR genes), cultured at low density in fibronectin (Sigma Chemical, St Louis, MO)-coated flasks, and induced to differentiate in vitro into neurons, hepatocytes, and endothelium, as described previously. ${ }^{2} \mathrm{~A}$ single MAPC-derived clone stably expressing DsRed2 and firefly luciferase was prepared using Sleeping Beauty transposons, ${ }^{13}$ as we described. ${ }^{14}$

\section{Intravenous and intra-arterial infusion of MAPCs}

For intravenous injections, MAPCs were infused through the tail vein. For intra-arterial injections under general anesthesia, small midline upper abdominal incisions were made, and the caudal aspect of diaphragm was exposed. After direct visualization of the heart apex, intracardiac injections of $10^{6}$ MAPCs (in $10 \mu \mathrm{L}$ PBS) were made slowly across the diaphragm and the left cardiac ventricular wall.

\section{Flow cytometry}

Single-cell suspensions of MAPCs were prepared in buffer (PBS $+2 \%$ bovine serum $+0.15 \%$ sodium azide). Pelleted cells were incubated for 15 minutes at $4{ }^{\circ} \mathrm{C}$ with $0.4 \mu \mathrm{g}$ anti- $\mathrm{Fc}$ receptor monoclonal antibody (mAb; clone $2.4 \mathrm{G} 2$, rat $\mathrm{IgG}_{2 \mathrm{~b}}$ ) to prevent $\mathrm{Fc}$ binding. Flow cytometry was performed using directly conjugated (fluorescein isothiocyanate [FITC] or phycoerythrin [PE]) mAbs to assess cell-surface antigen expression of MAPCs before and after 24 hours of stimulation with $1000 \mathrm{U}$ IFN- $\gamma / \mathrm{mL}$ (R\&D Systems, Minneapolis, MN). Optimal concentrations of directly conjugated mAbs were added to a total volume of 100 to $130 \mu \mathrm{L}$ and were incubated for 1 hour at $4^{\circ} \mathrm{C}$. The following $\mathrm{mAbs}$, obtained from PharMingen (San Diego, CA), were used: anti-H $2^{\mathrm{b}}$-specific $\mathrm{mAb}$ (clone EH-144, mouse $\mathrm{IgG}_{2 \mathrm{a}}$ ), anti-IA ${ }^{\mathrm{b}}$-specific $\mathrm{mAb}$ (clone AF6-120.1, mouse $\mathrm{IgG}_{2 \mathrm{a}}$ ), anti-CD80-specific $\mathrm{mAb}$ (clone 16-10A1, hamster $\mathrm{IgG}_{2}$ ), anti-CD86specific $\mathrm{mAb}$ (clone GL1, rat $\mathrm{IgG}_{2 \mathrm{a}}$ ), anti-ICAM-1-specific mAb (clone 3E2, hamster $\mathrm{IgG}_{1}$ ), and anti-CD40-specific mAb (clone HM40-3, hamster $\mathrm{IgM}_{\mathrm{K}}$ ). All samples were analyzed on a FACScalibur (Becton Dickinson, Palo Alto, CA) using CellQuest software (BD Biosciences, San Jose, CA). Forward scatter and 90-degree side scatter were used to identify and gate live MAPC populations. At least 10000 events were examined.

\section{Mixed lymphocyte reaction culture}

To measure the potential of MAPCs to stimulate allogeneic T-cell responses, we prepared purified $\mathrm{CD}^{+}{ }^{+} \mathrm{T}$ cells or whole $\mathrm{T}$ cells from single-cell suspensions of axillary, inguinal, and mesenteric lymph node cells isolated from BALB/c mice. Lymph node cells were depleted of NK cells for all cell preparations and CD8 ${ }^{+} \mathrm{T}$ cells (hybridoma 2.43, rat IgG2b; provided by Dr David Sachs, Charlestown, MA) for CD4 ${ }^{+}$cell preparations by coating with monoclonal antibodies and passage through a goat anti-mouse and goat anti-rat immunoglobulin-coated column (Cedarlane Laboratories, Hornby, ON, Canada). Purified T cells ( $10^{5}$ cells $)$ and $10^{3}$ to $10^{5}$ irradiated (3000 cGy by cesium ${ }^{137} \mathrm{Cs}$ irradiation) C57BL/6 MAPCs or MAPC DLs were cultured in 96-well, round-bottom plates at $37^{\circ} \mathrm{C}$ and $10 \%$ $\mathrm{CO}_{2}$ for the indicated time in DMEM (BioWhittaker, Walkersville, MD) containing 10\% FCS (Hyclone, Logan, UT), $50 \mathrm{mM}$ 2-mercaptoethanol (2-ME; Sigma), $10 \mathrm{mM}$ HEPES ( $N$-2-hydroxyethylpiperazine- $N N^{\prime}-2$ ethanesulfonic acid) buffer, $1 \mathrm{mM}$ sodium pyruvate (Life Technologies, Grand Island, NY), amino acid supplements (1.5 mM L-glutamine, Larginine L-asparagine; Sigma), and antibiotics (100 U/mL penicillin, 100 $\mathrm{mg} / \mathrm{mL}$ streptomycin; Sigma). Some MAPCs were pretreated with $1000 \mathrm{IU}$ IFN- $\gamma / \mathrm{mL}$ for 48 hours before the assay was initiated. Irradiated T-celldepleted splenocytes were prepared from C57BL/6 mice as a positive control for T-cell proliferation. For secondary cultures, $\mathrm{T}$ cells were recovered after 96 hours of culture, extensively washed, and restimulated with irradiated allogeneic B6 splenocytes, MAPCs, or MAPC DLs. On the indicated day of culture, each well was pulsed with tritiated thymidine $(1 \mu \mathrm{Ci}[0.037 \mathrm{MBq}] /$ well) (Amersham Life Sciences, Buckinghamshire, United Kingdom) for 18 hours before harvest and counted in the absence of scintillation fluid on a $\beta$-plate reader (Packard Instrument, Meriden, CT). At least 4 wells were analyzed per group.

\section{Susceptibility of MAPCs to NK-cell-mediated lysis}

To increase NK-cell number and activity, we gave B6 mice intraperitoneal injections of poly I:C (120 $\mu \mathrm{g} /$ mouse). After 48 hours, splenocytes were harvested. Target cells (Yac-1 cells, positive control for NK lysis; or MAPCs) were loaded with chromium ${ }^{51} \mathrm{Cr}$ an hour before the experiment and were washed 3 times, as described previously. ${ }^{15}$ Chromium-labeled MAPCs or Yac-1 cells (5000/well) were mixed with splenocytes from poly I:C-injected mice at various ratios (200:1-0.8:1) in a 96-well plate. Cells were incubated at $37^{\circ} \mathrm{C}$ for 4 hours and pelleted by centrifugation ( 5 minutes at $500 \mathrm{~g}$ ). To test the cytolytic potential of cells, $\gamma$ radioactivity was measured in the supernatant and expressed as counts per minute (cpm). Three assays were performed. Relative target cell lysis was calculated as: (sample $\mathrm{cpm}-$ spontaneous release)/(maximum release - spontaneous release) $\times 100 \%$.

\section{In vivo imaging of MAPCs}

Thirty days after the infusion of MAPCs, mice were anesthetized with pentobarbital (Nembutal; Abbott Laboratories, Abbott Park, IL) $(0.1 \mathrm{~mL} / 10$ mg body weight), and the abdomen and chest were shaved. Luciferin stock (30 $\mathrm{mg} / \mathrm{mL}$; Xenogen, Alameda, CA) was injected into the mice at 150 $\mathrm{mg} / \mathrm{kg}$ intraperitoneally. A grayscale reference image was taken of the position of the mice before luciferase activity was assessed. Bioluminescent signals were assessed at 5 minutes after luciferin injection at an integration time of 2 minutes with an in vivo imaging system that uses a cooled charge-coupled device (CCD) camera (IVIS100; Xenogen). Pseudocolor 
images representing the bioluminescent signal intensity (blue is the least intense and red is the most intense) were superimposed over the grayscale reference image. Scales for the pseudocolor intensity plots are displayed with the images.

\section{In vitro quantification of luciferase expression}

Tissue homogenates of lung specimens were harvested by centrifugation, mixed with $10 \mu \mathrm{L}$ luciferin stock ( $30 \mathrm{mg} / \mathrm{mL}$; Xenogen), and assayed immediately for bioluminescence activity on a Chameleon 425-100 Multilabel Counter (Hidex, Turku, Finland). Average relative luminescence values were expressed as counts per second and normalized to total protein (Dojindo Molecular Technologies, Gaithersburg, MD).

\section{Localization and differentiation of MAPCs in tissues}

Tissue specimens of the recipient animals were cryopreserved in optimal cutting temperature (OCT) medium (Sakura Finetek, Torrance, CA) at $-80^{\circ} \mathrm{C}$. Six-micrometer-thick fresh-frozen sections were mounted on glass slides, fixed in acetone for 10 minutes at room temperature, and incubated in normal donkey serum (Jackson ImmunoResearch, West Grove, PA) for 20 minutes. Cryosections were stained with nuclear stain 4',6-diamidino-2phenylindole (SlowFade Gold antifade reagent with DAPI; Molecular Probes, Eugene, OR) and examined for native fluorescence of DsRed 2 by confocal fluorescence microscopy (Olympus Flowview 500I; Olympus Optical, Tokyo, Japan). Images were acquired with Flowview 3.2 software (Olympus) and then processed with Adobe Photoshop CS8.0 (Adobe Systems, San Jose, CA). To assess MHC class I expression in donor cells, tissue sections were also stained with biotinylated primary $\mathrm{H} 2^{\mathrm{b}}$ antibody at a 1:100 concentration (BD PharMingen) and were incubated for 1 hour at room temperature. Slides were washed twice in PBS and stained using immunodetection with fluorescein (Vector M.O.M.; Vector Laboratories, Burlingame, CA) according to the manufacturer's instructions. Slides were examined using confocal fluorescence microscopy under $20 \times / 0.7$ and $40 \times / 0.9$ oil-immersion objective lenses (Olympus).

\section{Data analysis}

Differences between measurements were assessed using the Fisher exact test, with $P$ values below .05 considered significant.

\section{Results}

\section{MAPCs as targets of T- and NK-cell immune responses} as assessed in vitro

Immunophenotyping revealed that B6 MAPCs were low/negative for MHC class I and class II, costimulatory molecules (CD80, CD86, CD40), and the adhesion molecule ICAM-1 (CD54) (Figure 1A). Upon 24-hour stimulation with IFN- $\gamma$, MHC class I and ICAM-1 expression were up-regulated from $9.3 \%$ to $99 \%$ and from $2.9 \%$ to $35.9 \%$, respectively. Expression of MHC class II, CD80, CD86, and CD40 remained low. Low expression of molecules required for optimal T-cell responses suggested that MAPCs would be poor targets for $\mathrm{T}$ cells and good targets for NK-mediated elimination. To determine whether MAPCs could stimulate T cells, mixed lymphocyte reaction (MLR) assays were performed using $\mathrm{BALB} / \mathrm{c} \mathrm{CD} 4^{+} \mathrm{T}$ cells or $\mathrm{BALB} / \mathrm{c} \mathrm{CD} 4^{+}$plus $\mathrm{CD} 8^{+} \mathrm{T}$ cells as responders and B6 MAPCs as stimulators. Neither untreated MAPCs nor MAPCs pretreated with IFN- $\gamma$ for 48 hours stimulated $\mathrm{CD}^{+}{ }^{+}$(Figure 1B) or whole T-cell alloresponses (Figure 1C) in vitro. Proliferative T-cell responses to allogeneic MAPCs or MAPC DLs remained low even when the responder-stimulator ratio was increased as high as 1:1 (Figure 1D) or after repeated stimulation of $\mathrm{T}$ cells with allogeneic MAPCs or MAPC DLs (Figure 1E). To determine whether MAPCs could serve as targets of NK-mediated cytolysis, splenocytes from poly I:C (an inducer of NK activity)-treated B6 mice were mixed with Yac-1 $\left(\mathrm{H} 2^{\mathrm{a}}\right)$, an NK-sensitive target, or MAPCs in a 4-hour chromium release assay. Effector-target cell ratios indicated that MAPCs were susceptible to NK lysis but less so than Yac-1 cells (Figure 1F).

\section{In vivo immune resistance to MAPCs}

To assess in vivo immune responses to MAPCs, MAPCs were infused into mice with varying degrees of immune competence. MAPCs were nucleoporated with Sleeping Beauty transposon constructs to permit the expression of DsRed2 and firefly luciferase, yielding doubly transgenic MAPC DLs (DsRed2+ $2^{+}$luciferase $^{+}$), as previously described. ${ }^{14}$ To sequentially follow the homing, migration, and persistence of MAPCs in live animals in vivo, whole body imaging (WBI) was performed using luciferasemediated bioluminescence imaging (BLI). One million MAPC DLs were injected intravenously into adult immune competent B6 or T- and B-cell-deficient B6 Rag2-/- mice. Additional cohorts of B6 or B6 Rag2 ${ }^{-1-}$ mice were given anti-NK1.1 mAb to deplete NK cells. These data were compared with those of B6 Rag2/IL$2 \mathrm{R} \mathrm{c}^{-1-}$ mice that lack T, B, and NK cells.

Sequential BLI analysis was performed in 5 mice per group per time point on days 4,14 , and 30 after MAPC DL infusion. In B6 mice, MAPC DLs were detected in the thorax and the injection site (likely around the tail vein) on day 4 but not on day 14 or day 30 (data not shown). In Rag2 $2^{-1-}$ mice, MAPC DLs were detected throughout the 30-day period (B6 vs $\operatorname{Rag} 2^{-1-}$ at day 30: 34 vs 180 photons $/ \mathrm{s}$ per $\mathrm{cm}^{2}$, respectively; $P=.044$; Figure 2 ). Although NK depletion did not substantially increase MAPC DL number by BLI quantification in B6 mice (B6 without vs with NK depletion: 34 vs 40 photons/s per $\mathrm{cm}^{2}$, respectively; $\left.P=.198\right)$, it did in $\operatorname{Rag} 2^{-l-}$ mice by day 30 (B6 with NK depletion vs Rag2 ${ }^{-1-}$ with NK depletion: 40 vs 584 photons/s per $\mathrm{cm}^{2}$, respectively; $P=.004$; Figure 2). Rag2 ${ }^{-1-}$ mice, which have higher NK activity than B6 mice ${ }^{16}$ showed low levels of MAPC DL engraftment unless they were in vivo depleted of $\mathrm{NK}$ cells $\left(\operatorname{Rag} 2^{-1-}\right.$ without vs with $\mathrm{NK}$ depletion: 180 vs 584 photons/s per $\mathrm{cm}^{2}$, respectively; $P=.014$; Figure 2). In Rag2/IL-2R $\gamma \mathrm{c}^{-1-}$ mice, persistence of MAPC DLs was similar to that in $\operatorname{Rag} 2^{-1-}$ with NK depletion (data not shown). Collectively, these data indicate that endogenous NK cells resist MAPC DLs.

\section{Intra-arterial infusion of MAPCs results in superior biodistribution}

Because most of the bioluminescence of infused MAPC DLs was detected over the upper thorax, we reasoned that the capture of MAPCs in pulmonary vasculature after intravenous delivery may decrease the actual MAPC cell dose delivered to other visceral organs. This might have been because of the adhesion properties of MAPCs or their size (MAPCs measure 10-12 $\mu \mathrm{M}$ in diameter) in combination with the anatomic location of the lungs as the first capillary bed MAPCs encounter after intravenous infusion. To compare the biodistribution of MAPCs after intravenous and intra-arterial delivery, MAPC DLs $\left(10^{6}\right)$ were infused through the tail vein or through the left cardiac ventricle into Rag-2/IL$2 \mathrm{R} \gamma \mathrm{c}^{-1-}$ mice (3 mice per group per time point), which were permissive hosts for MAPC persistence. After intra-arterial delivery, WBI showed BLI signals distributed throughout the whole body at day 4 (Figure 3A) and to the thoracoabdominal area, face, and paws at day 30 (Figure 3B), in contrast to primarily thoracic BLI signals after intravenous delivery (Figure 2). Quantitatively, 
A

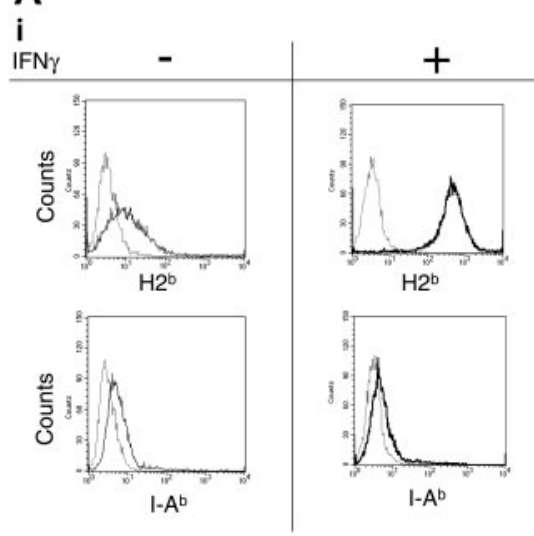

B

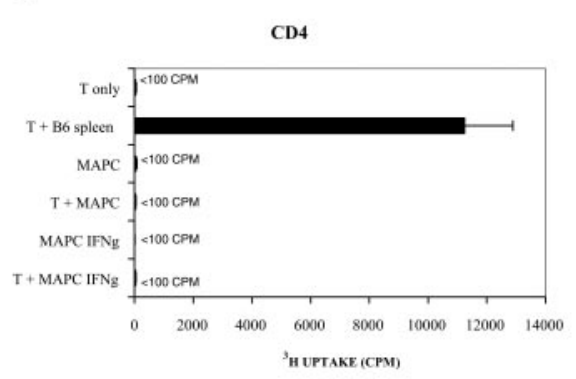

ii

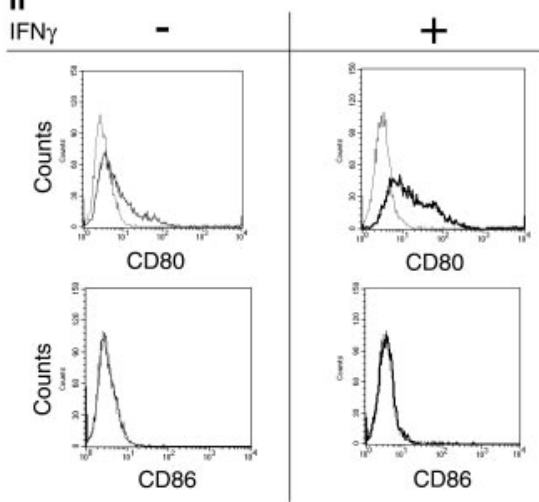

C

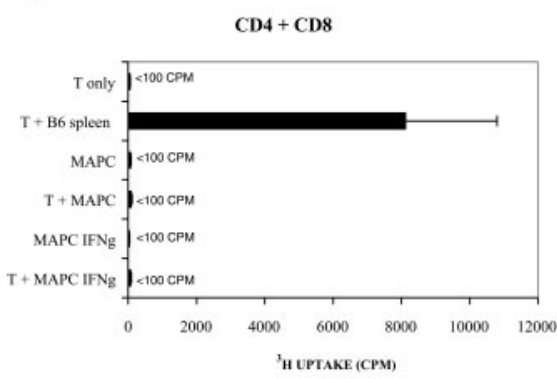

iii
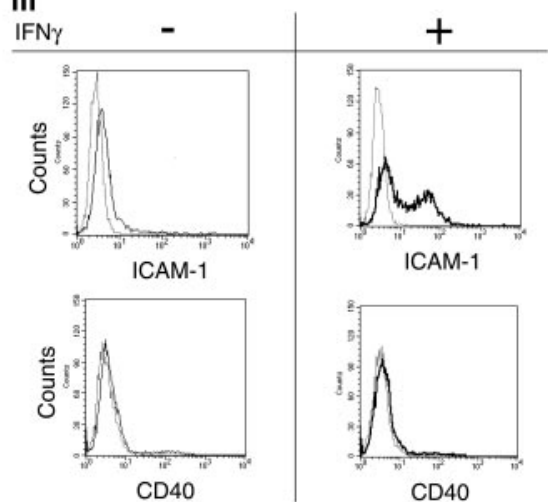

CD40

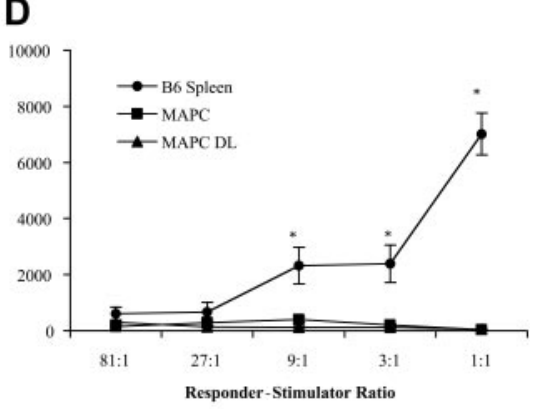

E

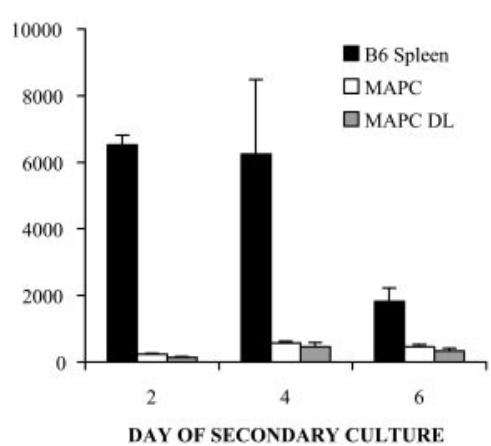

$\mathbf{F}$

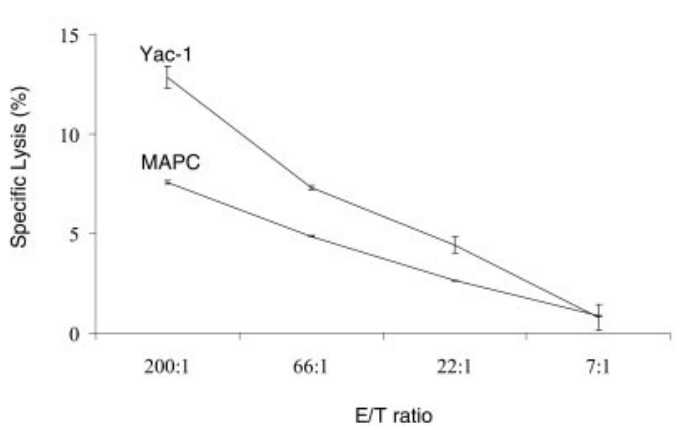

Figure 1. MAPCs do not stimulate T-cell responses and are susceptible to NK-cell-mediated lysis. (A) MAPCs are low/negative for MHC class I and class II, CD80 (B7-1), CD86 (B7-2), ICAM-1, and CD40. Upon 24 hours stimulation with $1000 \mathrm{IU} / \mathrm{mL}$ IFN- $\gamma$, MHC class I and ICAM-1 expression was up-regulated, whereas the expression of MHC class II, CD80, CD86, and CD40 remained low. Representative histograms of experiments performed 3 times are shown. (dashed line) Irrelevant antibody. (continuous line) Antibody to antigen indicated in each panel. (B) $1 \times 10^{5} \mathrm{BALB} / \mathrm{c} \mathrm{CD} 4^{+}$T cells/well or (C) $1 \times 10^{5} \mathrm{BALB} / \mathrm{c}$ CD4 ${ }^{+}$plus CD8 ${ }^{+}$T cells/well and irradiated, untreated B6 MAPCs or irradiated MAPCs $\left(10^{4} /\right.$ well) that were pretreated with $1000 \mathrm{IU} I F N-\gamma / \mathrm{mL}$ for 48 hours were mixed in T-cell proliferation assays. In some wells, T cells were cultured alone or with irradiated T-cell-depleted B6 splenocytes (105/well). T-cell proliferation was measured by ${ }^{3} \mathrm{H}$-thymidine uptake on day 5 and is expressed as mean $\pm \mathrm{SEM}$. (D) $10^{5} \mathrm{BALB} / \mathrm{C}$ $\mathrm{CD}^{+}$plus $\mathrm{CD}^{+} \mathrm{T}$ cells per well were cultured with the indicated number of allogeneic splenic stimulators, MAPCs, or MAPC DLs per well. Mean background counts for $10^{5}$ irradiated B6 splenocytes, MAPCs, or MAPC DLs were 37, 362, and $274 \mathrm{cpm}$, respectively (data not shown). (E) After a 96-hour primary MLR culture with irradiated B6 splenic stimulators or MAPCs, $10^{5} \mathrm{BALB} / \mathrm{C}$ CD4 ${ }^{+}$plus CD8 ${ }^{+}$T-cell responders/well were restimulated with $4 \times 10^{4}$ freshly prepared B6 splenic stimulators, MAPCs, or MAPC DLs per well. Mean background counts of $4 \times 10^{4}$ irradiated B6 splenocytes, MAPCs, or MAPC DLs were 82,434 , and $507 \mathrm{cpm}$, respectively (data not shown). (F) To determine whether MAPCs are susceptible targets for NK-cell-mediated killing, splenocytes from poly I:C-treated B6 mice were mixed with Yac-1 cells or with MAPCs in a chromium release assay. Effector-target cell ratios showed that MAPCs are a target of NK lysis in vitro.

BLI signals were higher at day 4 and day 30 after intra-arterial delivery than were signals observed after intravenous infusion of the same dose of MAPC DLs (on day 4 after intra-arterial delivery, mean BLI was 27-fold higher than after intravenous mean BLI; $P=.004$; on day 30 after intra-arterial delvery, mean BLI was 4-fold higher than after intravenous mean BLI; $P=.008$; data not shown and Figure 3).

To obtain an assessment of tissue-specific engraftment of donor MAPCs, tissue homogenates of lung, liver, kidney, brain, intestine, spleen, and bone marrow were examined for luciferase activity at day 30 after intravenous or intra-arterial MAPC DL infusion. Lung luminescence after intra-arterial infusion was above background but was significantly lower than lung luminescence after intravenous delivery (14 081 photons/s, range, 3921-15 564 photons/s; vs 205 photons/s, range, 131-261 photons/s; $P=.032$; Figure 3C). After intravenous administration, other tissues tested were below the limits of detection. In contrast, tissue luminescence was significantly higher after intra-arterial delivery than the same tissues after intravenous delivery: liver (197 photons/s; range, 111-252 photons/s; $P=.05)$, kidney (6197 photons/s; range, 1221-10 449 photons/s; $P=.044$ ), and brain (344 photons/s; range, 297-379 photons/s; $P=.004$; Figure 3C). Intra-arterial 

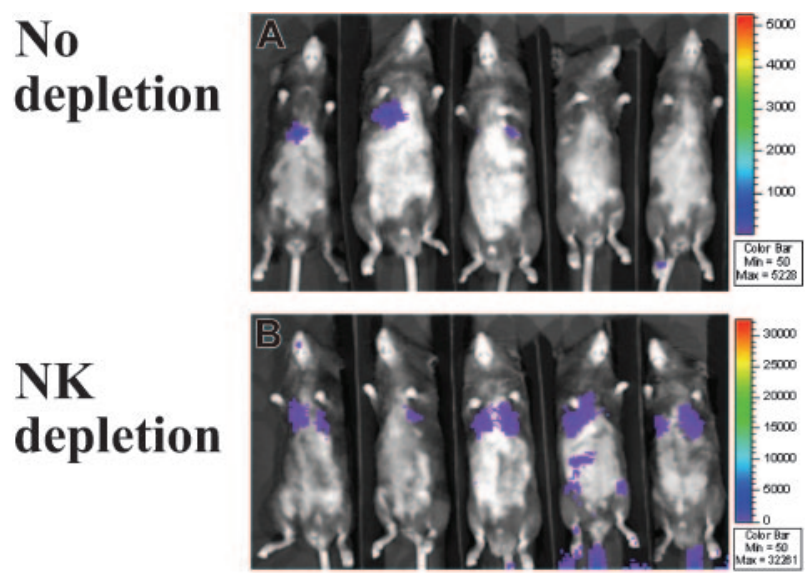

Figure 2. Immune resistance to MAPC in Rag2-/- mice. (A) With the use of WBI of firefly luciferase bioluminescence, the biodistribution of donor MAPC DLs was monitored in real time. In T- and B-cell-deficient Rag2 ${ }^{-1-}$ mice $(n=5)$, MAPC DLs were detected throughout the 30-day period and even more so $(B)$ in the mice given the anti-NK1.1 monoclonal antibody $(n=5)$. MAPC DL-derived BLI signals were observed predominantly over the area of upper thorax.

delivery was also higher in intestine, spleen, and BM, though these data did not reach statistical significance. These findings correlate with the WBI data from the same animals (Figure 3A-B) and show that, when compared with IV delivery, intra-arterial infusion results in much more diverse homing of MAPC DLs.

\section{Total body irradiation overcomes MAPC resistance}

Given that a long-term goal of these investigations is to use MAPCs clinically for the treatment of tissue injury as occurs in allogeneic hematopoietic cell transplantation, we sought to determine whether MAPC DLs can persist under these conditions. B6 mice were lethally irradiated and given B10.BR BM cells intravenously, along with $10^{6}$ MAPC DLs infused intravenously or intra-arterially. Bioluminescence signals from MAPCs were detected over the chest after intravenous administration (Figure 4A) and over the chest, abdomen, head, and extremities of recipient mice from day 4 through day 30 (Figure 4B). Although day 4 mean BLI signals after intra-arterial MAPC DL infusion were not significantly different from mean BLI signals after intravenous infusion, day $30 \mathrm{BLI}$ signals after intra-arterial infusion were 22 -fold higher than after intravenous infusion $(P<.01)$. In addition, after intra-arterial administration, the BLI signals increased 3 -fold between days 4 and 30 ( $P<.01$; data not shown and Figure 4B).

Using measurements of luciferase activity in tissue homogenates, we assessed the engraftment of donor MAPCs in lung, liver, kidney, brain, intestine, spleen, and bone marrow on day 30 after intravenous or intra-arterial MAPC DL infusion. After intra-arterial delivery, tissue luminescence was found to be significantly higher than background (and than samples of same tissues after intravenous delivery) in spleen (2527 photons/s; range, 168-4631 photons/s; $P<.04$ ), kidney (56 689 photons/s; range, 177-108 402 photons/s; $P<.03$ ), and brain (2443 photons/s; range, 225-4669 photons/s; $P<.04$; Figure $4 \mathrm{C}$ ). After intra-arterial or intravenous delivery, luciferase activity was also detected in intestine, liver, and BM (intra-arterial vs intravenous: 2644 vs 154 photons/s, 12146 vs 131 photons/s, and 683 vs 619 photons/s in intestine, liver, and BM, respectively), though these differences were not statistically different between intravenous and intra-arterial delivery. These data are similar to those described for the Rag2/IL-2R $\gamma \mathrm{c}^{-1-}$ mice that were permissive for MAPC engraftment. Lung luminescence was above background but significantly lower after intra-arterial delivery than was lung luminescence after intravenous delivery (171 236 vs 1650 photons/s; $P<.01$; Figure 4C). Overall, MAPC DL engraftment was higher with MAPC DLs and allogeneic BM infusion than after infusion of MAPC DLs alone using either route of delivery (Figures 3, 4), indicating that conditioning for hematopoietic stem cell transplantation using TBI may override NK- and T-cellmediated resistance and may be advantageous to the long-term survival and widespread homing of MAPCs.

\section{Tissue localization of MAPC DLs in vivo}

To assess donor engraftment at the level of individual organs, visceral tissues and brain were collected at the end of experiments, 30 days after infusion of MAPC DLs. Tissue immunofluorescence revealed MAPC DL cells in all tissues examined in all animals except nonconditioned B6 wild-type mice after intravenous delivery. Donor MAPC DL-derived cells in various tissues were similar in quality but differed in quantity, which in turn correlated with
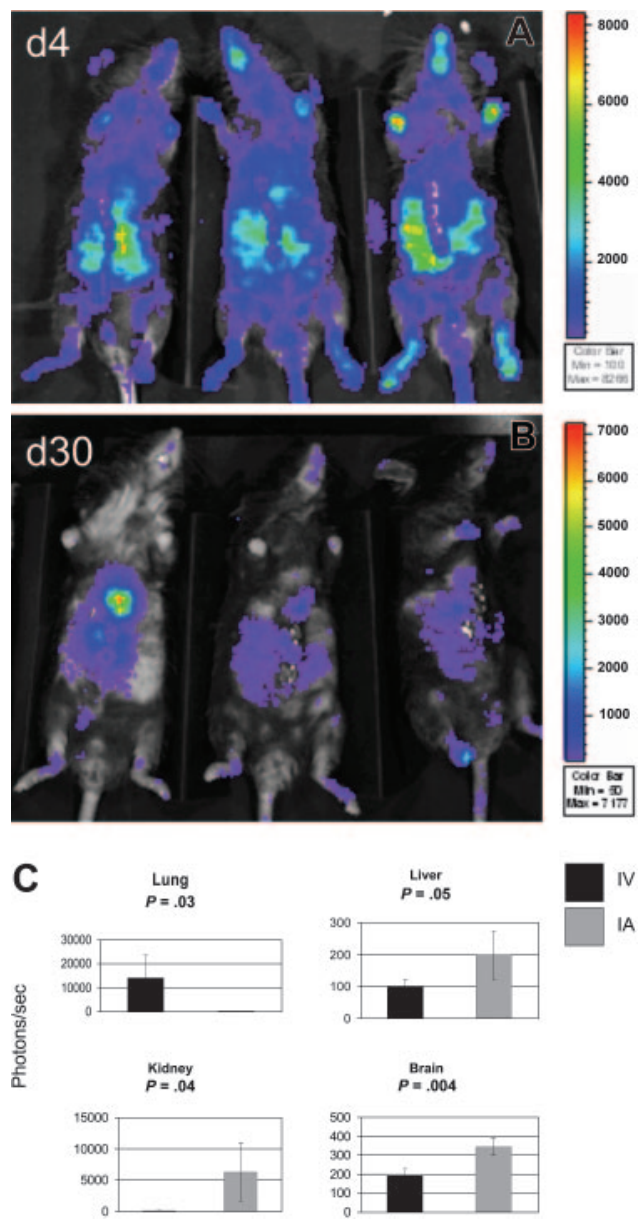

Figure 3. Intra-arterial infusion of MAPCs results in superior biodistribution. To assess the biodistribution of MAPCs after intra-arterial (IA) delivery, MAPC DLs $\left(10^{6}\right)$ were infused through the left cardiac ventricle $(n=3)$ into Rag2/IL-2R $\gamma \mathrm{c}^{-1-}$ mice. WBI is shown at 4 days $(A)$ and at 30 days $(B)$ after MAPC DL infusion. WBI showed $\mathrm{BLI}$ signals distributed throughout the whole body at day $4(\mathrm{~A})$ and the thoracoabdominal area, face, and paws at day $30(B)$, in contrast to mostly thoracic BLI signals after intravenous (IV) delivery (Figure 2B). (C) Tissue homogenates were examined for luciferase activity at day 30 after IV or IA MAPC DL infusion. Lung luminescence after IA infusion was above background but significantly less than lung luminescence after IV delivery $(P=.032)$. After IA delivery, tissue luminescence was significantly higher than in samples of the same tissues after IV delivery in liver $(P=.05)$, kidney $(P=.044)$, and brain $(P=.004)$. These findings show that, when compared with IV delivery, IA infusion results in more diverse homing of MAPC DLs. 

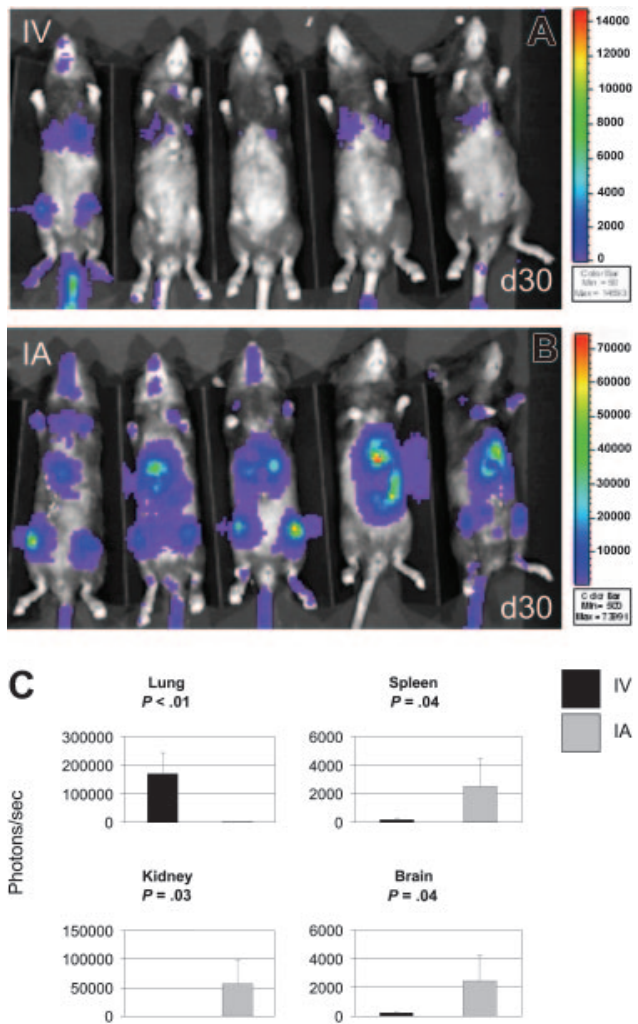

Figure 4. TBI overcomes MAPC resistance. To determine whether MAPC can persist under conditions of allogeneic hematopoietic stem cell transplantation, B6 mice were lethally irradiated and given B10.BR bone marrow intravenously with MAPC DLs infused intravenously (A) or intra-arterially (B). At day 30 after intravenous (IV) infusion, BLI signals from donor MAPC DLs were detected over thorax (A). After intra-arterial (IA) administration, bioluminescence was detected over the chest, abdomen, head, and extremities (B). (C) To quantify the engraftment of donor MAPCs, luciferase activity in tissue homogenates was determined at day 30 after cell infusions. After intra-arterial delivery, we detected tissue luminescence significantly higher than samples of the same tissues after intravenous delivery in spleen $(P=.04)$, kidney $(P=.03)$, and brain $(P=.04)$. Lung luminescence was above background but was significantly lower after intra-arterial delivery when compared with lung luminescence after intravenous delivery $(P=.01)$.

WBI and in vitro luciferase activity measurements (Figures 2-4). MAPC DL-derived cells were detected in the highest numbers in lung, kidney, and liver of irradiated B6 recipients of allogeneic BM and IA MAPC DLs (Figure 5A-C). In addition, in all tissues examined, including lung, donor MAPC DLs expressed MHC class I antigen (Figure 5D-F). These observations are critical because they provide evidence that transgenic MAPC DLs persist in vivo and they suggest that MAPC DLs may be functional in vivo.

\section{Discussion}

We show that MAPCs that are MHC class I low/negative are targets of NK lysis in vitro and in vivo and that the elimination of NK cells improves the persistence of reporter gene-labeled cells in T- and B-cell-deficient recipients. MAPCs that escape NK-mediated elimination persisted long-term. In vitro, undifferentiated MAPCs were found to express low levels of costimulatory and adhesion molecules and were poor stimulators of MLRs. In vivo, tissuelocalized MAPCs acquired MHC class I expression, and a role for the adaptive immune system was found in resisting reporter gene-expressing MAPCs that could be overcome by TBI conditioning. Route of delivery was found to be critical in directing MAPCs to the lung (intravenous) or toward other organs (intra-arterial).

MAPCs and other nonhematopoietic stem cells, such as mesenchymal stem cells (MSCs) ${ }^{17-19}$ — because of their ability to contribute to various tissues - may be advantageous in tissue repair after injury by conditioning chemoradiotherapy for hematopoietic stem cell transplantation. In contrast to hematopoietic progenitors, which have been associated with limited stem cell plasticity, ${ }^{20-23}$ undifferentiated MAPCs can differentiate into most, if not all, tissue types. ${ }^{2,5,24,25}$ Similar to MSCs, ${ }^{26}$ MAPCs were poor stimulators of an in vitro allogeneic MLR response. Consistent with the low level of MHC class I expression in undifferentiated MAPCs, NK cells were able to eliminate a large fraction of MAPCs in vivo.

Interestingly, despite the low or absent MHC class I expression on undifferentiated MAPCs, in vivo undifferentiated MAPCs were also resisted by the adaptive immune system. In vitro, IFN- $\gamma$ preincubation up-regulated MHC class I but not MHC class II expression. In vivo, tissue-localized MAPCs acquired MHC class I antigen expression even in mice to whom TBI was not given. Such induction of MHC class I expression could be attributed to local proinflammatory responses (eg, IFN- $\gamma$ production in vivo) as might occur in immune-deficient mice exposed to environmental pathogens that are not promptly removed or as a consequence of MAPC differentiation into a given tissue type in vivo. Despite the low level

Figure 5. MAPC DLs persist in tissues. Postmortem tissues analysis was performed on 2 to 4 representative animals from each cohort: intravenous delivery with and without NK depletion (B6, Rag2 ${ }^{-/-}$, Rag2/IL-2R $\gamma \mathrm{C}^{-/-}$), intra-arterial delivery (Rag2/IL-2R $\gamma \mathrm{c}^{-/-}$), and intravenous or intra-arterial MAPC DLs infused with B10.BR bone marrow into lethally irradiated B6 mice. MAPC DLderived cells were detected in multiple tissues. Shown here are donor MAPC DL-derived cells in the lung $(A)$, kidney $(B)$, and liver $(C)$ of the $B 6$ mouse, with the highest $\mathrm{BLI} 30$ days after intra-arterial infusion of MAPCs and intravenous infusion of allogeneic bone marrow (original magnification, $200 \times$ ). Donor MAPC DL-derived cells (thin arrows) appeared red as a result of native DsRed2 fluorescence, and nuclei were stained blue with DAPI. Donor MAPC DLs expressed MHC class I in multiple tissues. Shown here are lung cells expressing $\mathrm{H} 2 \mathrm{~b}$ (green; D) and DsRed2 (red; E) and the superimposition of the green and red pictures (donor MAPC DL cell, thick arrow; recipient cell, thin arrow; original magnification, $400 \times ; \mathrm{F})$.

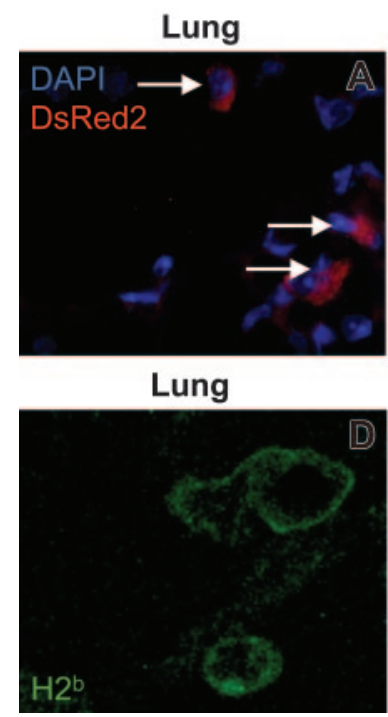

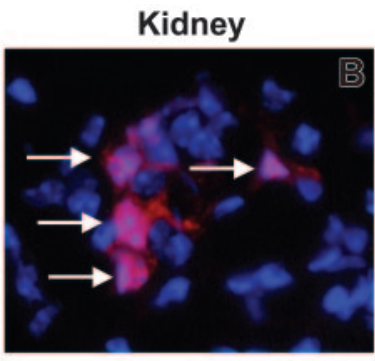

Lung

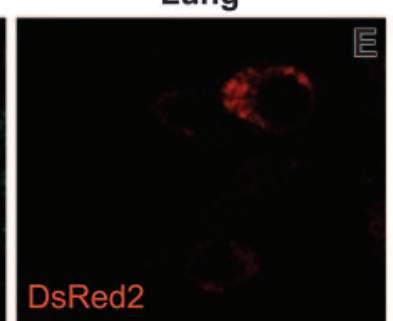

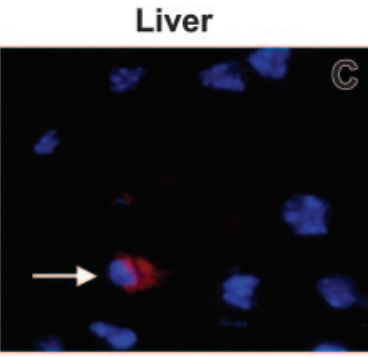

Lung

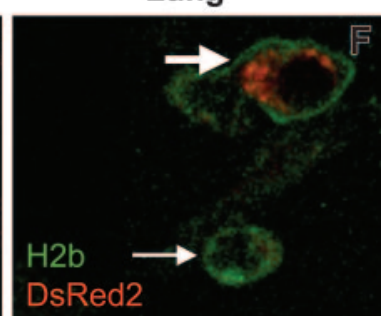


of costimulatory molecule expression even with IFN- $\gamma$ preincubation, undifferentiated MAPCs were resisted by the adaptive immune system. We speculate that this happens because these MAPCs acquire MHC class I antigens and express 4 distinct foreign antigens (DsRed2, luciferase, neomycin phosphotransferase, $\beta$ galactosidase). Other studies have demonstrated neomycin phosphotransferase induces cytolytic T-cell responses in vivo, ${ }^{27}$ and our own unpublished data (B.R.B., June 2005) indicate that up to a 10-fold higher dose of tumor cells expressing DsRed 2 is required to achieve equivalent lethality as the parental cell line, similar to results described for green fluorescence proteinexpressing tumor cells. ${ }^{28,29}$ Thus, undifferentiated MAPCs that are poorly immunogenic in vitro are converted to targets of the adaptive immune response in vivo under these conditions. MAPC homing and persistence may be increased during hematopoietic stem cell transplantation as a result of tissue injury, immune suppression (including reduction in T- and NK-cell numbers), up-regulation of MHC class I expression, or induction of cytokine and chemokine responses.

Because the production of autologous MAPCs can take several months, their use in clinical settings will be for diseases that do not require emergency treatment. Allogeneic MAPCs may represent a viable strategy on their own or as an adjunct to hematopoietic stem cell transplantation provided they are not rejected by the recipient.
Human MAPCs have characteristics similar to those of murine MAPCs. ${ }^{2,5,24,25}$ Therefore, optimal conditions for MAPC infusion can be identified in murine models, allowing the establishment of a basis for the use of human MAPCs in clinical settings.

Although intravenous delivery of MAPCs may be superior for their delivery to lung, intra-arterial administration may be applicable for the delivery of MAPCs to extrapulmonary organs. Thus, to promote tissue repair in various visceral organs, the site of infusion may have to be chosen accordingly to increase the usefulness of MAPCs for the recipient.

These data are the first to illustrate the immune responses to MAPCs. We conclude that MAPCs express low levels of MHC class I antigens and are targets of NK-mediated resistance and that TBI facilitates the persistence of MAPCs. In combination, our data suggest that the infusion of MAPCs along with BM may represent a new approach for the correction of genetic disorders and for reducing the conditioning regimen-related adverse effects of hematopoietic stem cell transplantation.

\section{Acknowledgments}

We thank Dr Jeffrey Miller for help with NK assays and Dr Patricia A. Taylor for critical reading of the manuscript.

\section{References}

1. Reyes M, Verfaillie CM. Characterization of multipotent adult progenitor cells, a subpopulation of mesenchymal stem cells. Ann N Y Acad Sci. 2001:938:231-233; discussion 233-235.

2. Jiang $Y$, Jahagirdar BN, Reinhardt RL, et al. Pluripotency of mesenchymal stem cells derived from adult marrow. Nature. 2002;418:41-49.

3. Jiang $Y$, Vaessen B, Lenvik T, Blackstad M, Reyes M, Verfaillie CM. Multipotent progenitor cells can be isolated from postnatal murine bone marrow, muscle, and brain. Exp Hematol. 2002; 30:896-904.

4. Jiang $Y$, Henderson D, Blackstad M, Chen A, Miller RF, Verfaillie CM. Neuroectodermal differentiation from mouse multipotent adult progenitor cells. Proc Natl Acad Sci U S A. 2003;100(suppl 1):11854-11860

5. Schwartz RE, Reyes M, Koodie L, et al. Multipotent adult progenitor cells from bone marrow differentiate into functional hepatocyte-like cells. J Clin Invest. 2002;109:1291-1302.

6. Lanier LL. NK cell recognition. Annu Rev Immunol. 2005;23:225-274.

7. Raulet DH. Interplay of natural killer cells and their receptors with the adaptive immune response. Nat Immunol. 2004;5:996-1002.

8. Seino K, Taniguchi M. Functional roles of NKT cell in the immune system. Front Biosci. 2004;9: 2577-2587.

9. Auletta JJ, Devecchio JL, Ferrara JL, Heinzel FP. Distinct phases in recovery of reconstituted innate cellular-mediated immunity after murine syngeneic bone marrow transplantation. Biol Blood Marrow Transplant. 2004;10:834-847.

10. Murphy WJ, Kumar V, Bennett M. Acute rejection of murine bone marrow allografts by natural killer cells and T cells: differences in kinetics and target antigens recognized. J Exp Med. 1987;166:14991509.

11. Bevis BJ, Glick BS. Rapidly maturing variants of the Discosoma red fluorescent protein (DsRed). Nat Biotechnol. 2002;20:83-87.

12. Edinger M, Hoffmann $\mathrm{P}$, Contag $\mathrm{CH}$, Negrin RS. Evaluation of effector cell fate and function by in vivo bioluminescence imaging. Methods. 2003; 31:172-179.

13. Ivics $Z$, Hackett PB, Plasterk RH, Izsvak Z. Molecular reconstruction of Sleeping Beauty, a Tc1like transposon from fish, and its transposition in human cells. Cell. 1997;91:501-510.

14. Tolar J, Osborn M, Bell S, et al. Real-time in vivo imaging of stem cells following transgenesis by transposition. Mol Ther. 2005;12:42-48.

15. Kim S, lizuka K, Kang HS, et al. In vivo developmental stages in murine natural killer cell maturation. Nat Immunol. 2002;3:523-528.

16. Prlic M, Blazar BR, Khoruts A, Zell T, Jameson SC. Homeostatic expansion occurs independently of costimulatory signals. J Immunol. 2001; 167:5664-5668.

17. Pittenger MF, Mackay AM, Beck SC, et al. Multilineage potential of adult human mesenchymal stem cells. Science. 1999;284:143-147.

18. Peister A, Mellad JA, Larson BL, Hall BM, Gibson LF, Prockop DJ. Adult stem cells from bone marrow (MSCs) isolated from different strains of inbred mice vary in surface epitopes, rates of proliferation, and differentiation potential. Blood. 2004; 103:1662-1668.

19. Baksh D, Song L, Tuan RS. Adult mesenchymal stem cells: characterization, differentiation, and application in cell and gene therapy. J Cell Mol Med. 2004;8:301-316.

20. Wagers AJ, Sherwood RI, Christensen JL, Weiss man IL. Little evidence for developmental plasticity of adult hematopoietic stem cells. Science. 2002;297:2256-2259.

21. Lagasse E, Connors H, Al-Dhalimy M, et al. Purified hematopoietic stem cells can differentiate into hepatocytes in vivo. Nat Med. 2000;6:12291234.

22. Krause DS, Theise ND, Collector MI, et al. Multiorgan, multi-lineage engraftment by a single bone marrow-derived stem cell. Cell. 2001;105:369377.

23. Borue X, Lee S, Grove J, et al. Bone marrow-derived cells contribute to epithelial engraftment during wound healing. Am J Pathol. 2004;165: 1767-1772.

24. Reyes M, Dudek A, Jahagirdar B, Koodie L, Marker $\mathrm{PH}$, Verfaillie CM. Origin of endothelial progenitors in human postnatal bone marrow. J Clin Invest. 2002;109:337-346.

25. Zhao LR, Duan WM, Reyes M, Keene CD, Verfaillie CM, Low WC. Human bone marrow stem cells exhibit neural phenotypes and ameliorate neurological deficits after grafting into the ischemic brain of rats. Exp Neurol. 2002;174:11-20.

26. Mahmud N, Pang W, Cobbs C, et al. Studies of the route of administration and role of conditioning with radiation on unrelated allogeneic mismatched mesenchymal stem cell engraftment in a nonhuman primate model. Exp Hematol. 2004; 32:494-501.

27. Berger C, Huang ML, Gough M, Greenberg PD, Riddell SR, Kiem HP. Nonmyeloablative immunosuppressive regimen prolongs in vivo persistence of gene-modified autologous T cells in a nonhuman primate model. J Virol. 2001;75:799-808.

28. Morris JC, Conerly M, Thomasson B, Storek J, Riddell SR, Kiem HP. Induction of cytotoxic Tlymphocyte responses to enhanced green and yellow fluorescent proteins after myeloablative conditioning. Blood. 2004;103:492-499.

29. Goto H, Yang B, Petersen D, et al. Transduction of green fluorescent protein increased oxidative stress and enhanced sensitivity to cytotoxic drugs in neuroblastoma cell lines. Mol Cancer Ther. 2003;2:911-917. 